\title{
Light element abundances in the young open clusters NGC 3293, NGC 4755 and NGC 6231: Tracers of stellar evolution ${ }^{\star}$
}

\author{
G. Mathys ${ }^{1}$, S. M. Andrievsky ${ }^{2,3}$, B. Barbuy ${ }^{2}$, K. Cunha ${ }^{4}$, and S. A. Korotin ${ }^{3,5}$ \\ 1 European Southern Observatory, Casilla 19001, Santiago 19, Chile \\ e-mail: gmathys@eso.org \\ 2 Universidade de São Paulo, CP 3386, São Paulo 01060-970, Brazil \\ e-mail: barbuy@orion.iagusp.usp.br; sergei@andromeda.iagusp.usp.br \\ 3 Department of Astronomy, Odessa State University, Shevchenko Park, 65014, Odessa, Ukraine \\ 4 Observatório Nacional, Rua General Cristino 77, CEP 20921-400, Rio de Janeiro, Brazil \\ e-mail: katia@maxwell.on.br \\ ${ }^{5}$ Odessa Observatory and Isaac Newton Institute of Chile, Odessa Branch, Ukraine \\ e-mail: serkor@skyline.od.ua
}

Received 19 November 2001 / Accepted 14 March 2002

\begin{abstract}
The abundances of He (LTE), C, N, and O (NLTE) were derived for 21 B stars in three young open clusters. Almost all the stars show subsolar CNO abundances. However, the mean oxygen abundance for each programme cluster appears to be in marginal agreement with the most recent revisions of the solar value. After consideration of the $\mathrm{CN}$ abundances in this sample, there is no clear evidence of internal mixing. Only three stars among the non-supergiants seem to show a nitrogen enhancement. Two of them have a fairly low projected equatorial velocity (admittedly, they may be rapid rotators seen pole-on); the third one is a definite fast rotator. In the lower gravity stars (three stars in this sample with $\log g<3.0$ ) some kind of mixing has apparently occurred. The supergiants do not differ significantly from the other programme stars in their respective helium contents. The mean helium abundance for each cluster is close to the standard value, $(\mathrm{He} / \mathrm{H}) \approx 11.0$.
\end{abstract}

Key words. open clusters and associations: individual: NGC 3293, NGC 4755, NGC 6231 - stars: abundances stars: early type

\section{Introduction}

Several aspects of the intermediate stages of the evolution of massive stars are still poorly understood. In particular, little is known about the possible occurrence of internal mixing in these stars. In massive stars, this process may manifest itself observationally through the appearance at the stellar surface of material that has been mixed in the interior, hence of products of the CNO-cycle. The presence of such material can be diagnosed from determinations of the abundances of carbon, nitrogen and oxygen in early B stars near the end of their main-sequence lifetimes and slightly evolved from the main-sequence, in young open clusters. Constraints on the mixing models can be derived through investigation of possible dependencies of these abundances on stellar parameters.

Lyubimkov (1984, 1989a, 1989b) claimed that relationships exist between the abundances of $\mathrm{He}, \mathrm{C}$ and $\mathrm{N}$ and stellar mass and age. More precisely, the abundances

Send offprint requests to: S. M. Andrievsky,

e-mail: scan@deneb.odessa.ua

* Based on spectra collected at the European Southern Observatory, La Silla, Chile, as part of programme 47.7-045. of He and $\mathrm{N}$ increase with the stellar age, while carbon abundances decrease. The rate at which helium and nitrogen are enhanced is proportional to the stellar mass. The sum of $\mathrm{C}$ and $\mathrm{N}$ abundances remains constant, as expected for CN-processed material. On the other hand, Gies \& Lambert (1992) carried out a comprehensive study of B supergiant and non-supergiant stars in order to check Lyubimkov's conclusions. They did not find clear evidence that nitrogen abundances progressively increase with stellar age. Nevertheless, they found evidence for the presence of $\mathrm{CN}$-cycled material in a few main-sequence stars. The atmospheres of all supergiant stars appeared to be enriched in nitrogen.

Andrievsky et al. (1999) derived the abundances of carbon and nitrogen in hot main-sequence stars using NLTE calculations. For almost all stars the $\mathrm{C}$ and $\mathrm{N}$ abundances appeared to be subsolar, but with a solar $\mathrm{C} / \mathrm{N}$ ratio, while for three stars a carbon deficiency was detected together with a nitrogen enhancement. This could be the signature of effective mixing in these three stars.

Recently, a thorough theoretical study on rotationally induced internal mixing and changes in the surface abundances of massive stars was performed by 


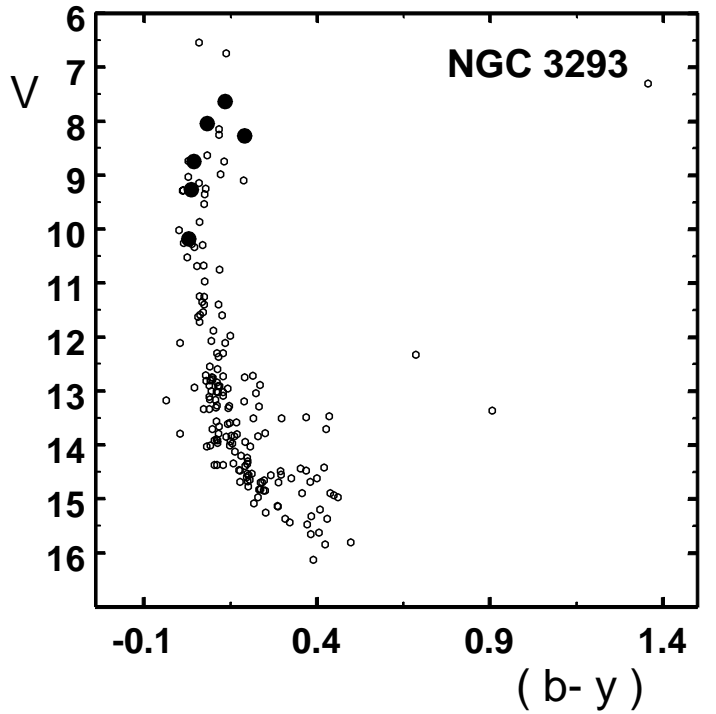

Fig. 1. Colour-magnitude diagram (CMD) for NGC 3293. Programme stars are shown as filled black circles. The photometric data are from Balona (1994).

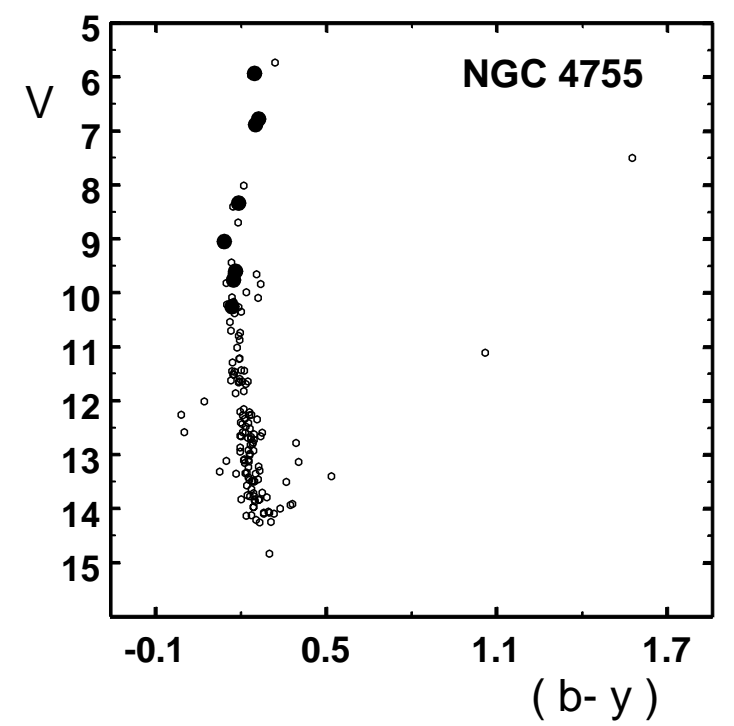

Fig. 2. CMD for NGC 4755. The photometric data are from Balona \& Koen (1994).

Heger \& Langer (2000). According to these authors rotational mixing is responsible for abundance anomalies found in stars with masses 10-20 $M_{\odot}$.

It should be noted that the CNO abundance anomalies, similar to those produced by rotational mixing, can also be caused by the mass transfer in close binary systems when the atmosphere of the accreting star is contaminated with the companion's material processed in the incomplete CNO cycle of hydrogen burning.

In this work, we present NLTE abundance analyses of a sample of hot stars in three young open clusters: NGC 3293, NGC 4755 and NGC 6231. In Sect. 2, the observations are described. The methods used for the analysis of the data are presented in Sect. 3. In Sects. 4, 5 and 6, the adopted stellar parameters, the derived elemental

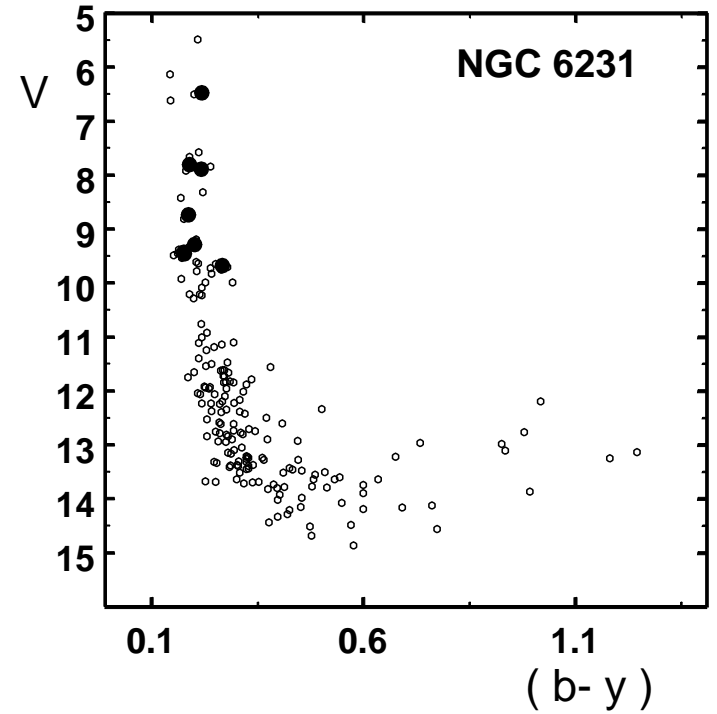

Fig. 3. CMD for NGC 6231. The photometric data are from Balona \& Laney (1995).

abundances, and their uncertainties are reported. Results are discussed in Sect. 7.

\section{Observations}

This study is based on observations carried out with the $3.6 \mathrm{~m}$ telescope of the European Southern Observatory at La Silla, Chile. Observations were carried out in May 1991 using the ESO Cassegrain Echelle Spectrograph CASPEC with the 52 lines $\mathrm{mm}^{-1}$ échelle grating and the blue-optimized cross-disperser grating. The detector was CCD \#16, a UV-flooded Tektronix with $512 \times 512$ pixels of $27 \mu \mathrm{m}$. The spectra cover the range 3900-5100 $\AA$ at a resolving power of 18000 . The reductions have been performed using the MIDAS echelle package. Details of the observations are given in Table 1 . Column 3 gives the heliocentric Julian date of mid-observation, and Col. 4, the total exposure time. The $S / N$ ratio per pixel given in the last column of the table has been computed from the measured rms in the continuum of the fully reduced spectrum.

The sample stars all appear in Mermilliod's (1999) lists of cluster members. This should ensure that, to the best of our present knowledge, all do indeed belong to their respective cluster. For NGC 4755, membership probabilities based on proper motions have actually been published by King (1981). This probability is above $90 \%$ for 6 of the 8 stars studied here, while it is $54 \%$ for NGC $4755-2$ and $58 \%$ for NGC $4755-18$. The programme stars have been selected so as to sample the widest possible range of masses at the top of the cluster sequence, taking into account the limitations set by the efficiency of the telescopeinstrument combination. Their locations in the colourmagnitude diagrams of the studied clusters are shown in Figs. 1 to 3. 
Table 1. Log of observations.

\begin{tabular}{rrcrr}
\hline \hline Star & $V$ & HJD & $\begin{array}{c}\text { Exp. } \\
(\min )\end{array}$ & $S / N$ \\
\hline \multicolumn{5}{c}{ NGC 3293 } \\
\hline 8 & 8.27 & 2448399.548 & 30 & 230 \\
13 & 10.18 & 2448400.530 & 105 & 270 \\
16 & 8.74 & 2448399.509 & 42 & 240 \\
18 & 9.27 & 2448400.588 & 45 & 260 \\
20 & 8.04 & 2448400.465 & 12 & 180 \\
22 & 7.63 & 2448399.471 & 18 & 100 \\
\hline & & NGC 4755 & & \\
\hline 2 & 5.93 & 2448398.565 & 12 & 180 \\
3 & 6.78 & 2448398.590 & 18 & 190 \\
5 & 8.33 & 2448398.622 & 36 & 260 \\
6 & 9.05 & 2448399.607 & 80 & 190 \\
7 & 9.76 & 2448398.716 & 160 & 130 \\
18 & 9.60 & 2448399.694 & 105 & 180 \\
106 & 6.88 & 2448400.714 & 9 & 220 \\
414 & 10.25 & 2448400.670 & 120 & 170 \\
\hline & \multicolumn{5}{c}{ NGC 6231} & & \\
\hline 161 & 7.89 & 2448400.871 & 18 & 200 \\
232 & 9.67 & 2448399.793 & 120 & 200 \\
286 & 9.43 & 2448399.870 & 75 & 220 \\
287 & 9.28 & 2448398.817 & 90 & 200 \\
292 & 8.73 & 2448398.873 & 45 & 180 \\
293 & 6.47 & 2448398.891 & 6 & 210 \\
323 & 7.80 & 2448398.909 & 24 & 170 \\
\hline & & & &
\end{tabular}

\section{Lines selected for analysis and calculations}

The great majority of the programme stars have reasonably sharp spectral lines that lend themselves to direct equivalent width measurements. From lines present in overlapping orders, we estimated the upper limit of the uncertainty in the measured equivalent widths to be approximately $10 \%$. The list of lines used in the analysis and their equivalent widths are gathered in Tables A.1, A.2 and A.3.

To represent the atmospheric structure of the studied stars, we used plane-parallel, LTE models interpolated from the grid of Kurucz (1992). The latter have been computed for three values of the microturbulence: 2,4 , and $8 \mathrm{~km} \mathrm{~s}^{-1}$. The model corresponding to the value of the microturbulence closest to the value derived from the present abundance study (see Sect. 4.2) was adopted whenever the microturbulence did not exceed $8 \mathrm{~km} \mathrm{~s}^{-1}$. For stars with larger microturbulence, grid models calculated with the maximum value of this parameter, $8 \mathrm{~km} \mathrm{~s}^{-1}$, were used. Some models (high temperature/low gravity) are not available in the grid, so they were computed using the ATLAS9 code.

\subsection{LTE determination of the helium abundance}

Helium abundances were determined through LTE analysis of HeI lines. For this purpose, we employed the WIDTH9 code of Kurucz. The oscillator strengths and damping constants were taken from CD-ROM 23 (Kurucz 1994).

\subsection{Non-LTE determination of the CNO abundances}

An updated version of Carlsson's (1986) NLTE code MULTI was used for the determination of elemental abundances of carbon, nitrogen and oxygen. The modifications of the code, atomic models and atomic level characteristics are given in detail in Korotin et al. (1999a, 1999b), Andrievsky et al. (1999), and Korotin et al. (1999c), as summarized below:

1. The atomic model of carbon used in the calculations includes 71 levels of CII, 12 levels of CIII, and the ground level of the $\mathrm{C}$ IV. The radiative transitions between the first 38 levels of $\mathrm{C}$ II, three C III levels, and the ground level of C IV were considered.

2. The model of the nitrogen atom consists of 109 levels: 3 ground levels of N I, 93 levels of N II with $L \leq 4$ and $n \leq 6,12$ levels of $\mathrm{N}$ III, and the ground state of N IV. Within the system of the nitrogen atom levels we considered the radiative transitions between the first 43 levels of N II, $5 \mathrm{~N}$ III levels, and the ground level of NiV.

3. The oxygen atomic model comprises 141 levels: 3 levels of O I, 132 levels of O II with $L \leq 5$ and $n \leq 8,5$ levels of OIII, and the ground state of O IV. In this system, we considered the radiative transitions between the first 49 levels of $\mathrm{O}$ II and the ground level of O III.

Our version of the MULTI code allows one to analyse the blends of lines of the same element. This option was used in cases where no single carbon lines are available in the spectrum (e.g., for NGC 4755-5).

\section{Stellar parameters}

\subsection{Effective temperatures and gravities of the programme stars}

Values of the effective temperature and gravity of the programme stars were derived from Strömgren photometry using the code written by T. T. Moon (based on the grid published in Moon \& Dworetsky 1985) as modified by Napiwotzki (1994). Complete information on the photometric data for the programme stars can be found in the SIMBAD database, or the WEBDA database (Mermilliod 1999). As can be seen in SIMBAD, many of the studied stars are suspected to be variable. To minimize the uncertainty introduced in temperature and gravity determinations by photometric variability, mean indices were determined using all the Strömgren colour index measurements available in the literature. In this averaging process, weights were assigned to the indices coming from different sources on the basis of the number of measurements of the considered index in that reference. For a few stars, data from various authors show rather significant differences. The resulting uncertainties in the derived atmospheric parameters will be further discussed in Sect. 6 . The adopted values of the mean photometric indices, and the resulting temperatures and gravities are given in Table 2 .

Note that there exist indications that some of our program stars may be binary systems. For example, in 
Table 2. Parameters of programme stars.

\begin{tabular}{|c|c|c|c|c|c|c|c|c|c|c|}
\hline Star & $\mathrm{DM}^{a}$ & $\begin{array}{l}\text { Spectral } \\
\text { type }\end{array}$ & $(b-y)$ & $m_{1}$ & $c_{1}$ & $\beta$ & $\begin{array}{l}T_{\text {eff }} \\
(\mathrm{K})\end{array}$ & $\log g$ & $\begin{array}{c}\xi_{\mathrm{t}} \\
\left(\mathrm{km} \mathrm{s}^{-1}\right)\end{array}$ & $\begin{array}{c}v \sin i \\
\left(\mathrm{~km} \mathrm{~s}^{-1}\right)\end{array}$ \\
\hline \multicolumn{11}{|c|}{ NGC $3293^{b}$} \\
\hline 8 & $-57^{\circ} 3540$ & B0.5 III & 0.192 & -0.018 & -0.019 & 2.570 & 26000 & 3.60 & 13 & 31 \\
\hline 13 & $-57^{\circ} 3522$ & $\mathrm{~B} 2 \mathrm{~V}$ & 0.030 & 0.069 & 0.050 & 2.620 & 24350 & 4.15 & 3 & 24 \\
\hline 16 & $-57^{\circ} 3500$ & $\mathrm{~B} 0.5 \mathrm{~V}$ & 0.045 & 0.047 & 0.015 & 2.592 & 25000 & 3.75 & 12 & 33 \\
\hline 18 & $-57^{\circ} 3524$ & B1 V & 0.038 & 0.052 & 0.038 & 2.606 & 24500 & 3.95 & 6 & 28 \\
\hline 20 & $-57^{\circ} 3523$ & B1 III & 0.084 & 0.018 & -0.023 & 2.575 & 25800 & 3.60 & 15 & 115 \\
\hline 22 & $-57^{\circ} 3506$ & B1 II & 0.135 & 0.012 & -0.021 & 2.564 & 24900 & 3.30 & 15 & 55 \\
\hline \multicolumn{11}{|c|}{ NGC $4755^{c}$} \\
\hline 2 & $-59^{\circ} 4555$ & B3 Ia & 0.248 & -0.030 & 0.130 & 2.551 & 15750 & 2.00 & 18 & 47 \\
\hline 3 & $-59^{\circ} 4566$ & B2 Ib & 0.263 & -0.054 & 0.125 & 2.568 & 17100 & 2.40 & 18 & 47 \\
\hline 5 & $-59^{\circ} 4552$ & B1 III & 0.192 & -0.024 & 0.027 & 2.588 & 25500 & 3.75 & 16 & 85 \\
\hline 6 & $-59^{\circ} 4564$ & B2 III & 0.142 & 0.003 & 0.062 & 2.605 & 24400 & 3.90 & 8 & 95 \\
\hline 7 & $-59^{\circ} 4528$ & B0.5 V & 0.174 & -0.007 & 0.048 & 2.601 & 25100 & 3.90 & 6 & 37 \\
\hline 18 & $-59^{\circ} 4553$ & B2 IV & 0.182 & 0.006 & 0.133 & 2.598 & 21500 & 3.40 & 0 & 30 \\
\hline 106 & $-59^{\circ} 4543$ & $\mathrm{~B} 1.5 \mathrm{Ib}$ & 0.251 & -0.055 & 0.025 & 2.559 & 21350 & 2.80 & 18 & 47 \\
\hline 414 & $-59^{\circ} 4535$ & B1 V & 0.169 & 0.022 & 0.139 & 2.632 & 22350 & 4.10 & 2 & 67 \\
\hline \multicolumn{11}{|c|}{ NGC $6231^{d}$} \\
\hline 161 & $-41^{\circ} 11050$ & O8.5 III & 0.217 & -0.031 & -0.032 & 2.585 & 30000 & 4.20 & 20 & 41 \\
\hline 232 & 521 & B0.5 V & 0.267 & -0.046 & 0.055 & 2.629 & 26470 & 4.60 & 8 & 45 \\
\hline 286 & 378 & B0.5 V & 0.176 & 0.009 & 0.073 & 2.627 & 24850 & 4.35 & 12 & 42 \\
\hline 287 & 403 & $\mathrm{~B} 1 \mathrm{~V}$ & 0.201 & 0.004 & 0.089 & 2.635 & 24300 & 4.45 & 2 & 8 \\
\hline 292 & $-41^{\circ} 11038$ & $09.5 \mathrm{~V}$ & 0.187 & -0.016 & -0.046 & 2.596 & 31700 & 4.60 & 20 & 83 \\
\hline 293 & $-41^{\circ} 11036$ & O9 Iab p & 0.219 & -0.025 & -0.097 & 2.552 & 32400 & 4.00 & 20 & 42 \\
\hline $323^{e}$ & 862 & O9 III & 0.190 & -0.024 & -0.068 & 2.587 & 33370 & 4.60 & 20 & 52 \\
\hline
\end{tabular}

${ }^{a}$ For those stars of NGC 6231 that do not have a DM number, the WEBDA number (Mermilliod 1999) is given as second identifier.

${ }^{b}$ Star numbering from Turner et al. (1980); MK types from Feast (1958), except for star 16 (Morgan et al. 1955).

${ }^{c}$ Star numbering from Arp \& van Sant (1958); MK types from Schild (1970), except for stars 2 (Walborn 1972 ), 7 and 414 (Feast 1963).

${ }^{d}$ Star numbering from Seggewiss (1968); MK types from Levato \& Malaroda (1980).

${ }^{e}$ Star 12 of Struve (1944)

a recent study, Garcia \& Mermilliod (2001) report that NGC 6231-161, 232, 286 and 287 are definite spectroscopic binaries; stars 161 and 232 probably have double-lined spectra. The same authors also conclude that the binarity percentage among the OB stars of NGC 6231 is very high, more than $82 \%$. Binarity can cause some ambiguity in photometric determinations of the stellar parameters, if the contribution of the secondary to the observed combined flux is significant (as is the case in particular for SB2s).

Another potential source of error in the estimation of the stellar parameters from Strömgren photometry is the possible presence of emission in the $\mathrm{H} \beta$ line, which could affect the $\beta$-index. For instance, we note that star NGC 4755-6 is reported as Be in the WEBDA database. It is quite possible that other targets could also be unrecognized Be stars. Therefore, the profile of $\mathrm{H} \beta$ in all the program stars was checked for appearance of emission features. The line seems to be purely in absorption in all stars, with the exception of NGC 6231-161, where it shows a small redshifted emission component.
In all cases where a sufficient number of $\mathrm{O}$ II and N II lines could be analyzed, the photometric $T_{\text {eff }}$ determination was refined spectroscopically, by requiring that the abundances derived from individual O II and N II lines do not depend on their lower level excitation potentials. As an example, in Fig. 4 we show such a plot for O II lines of the star NGC 3293-8.

\subsection{Microturbulent velocities}

The microturbulent velocities $\xi_{\mathrm{t}}$ for the programme stars were determined by requiring NLTE oxygen and nitrogen abundances derived from individual O II and N II lines to be independent from their equivalent widths. The number of O II lines measured in the spectra is sufficient for a reliable determination of $\xi_{\mathrm{t}}$ in all studied stars except for NGC 6231-161, 293, and 323. For the latter, the value of $\xi_{\mathrm{t}}$ estimated for NGC 6231-292 is adopted, based on the similarity of their spectral types. For some stars, the number of N II lines is also sufficient to derive a value of the microturbulence. In all cases, the results obtained from 


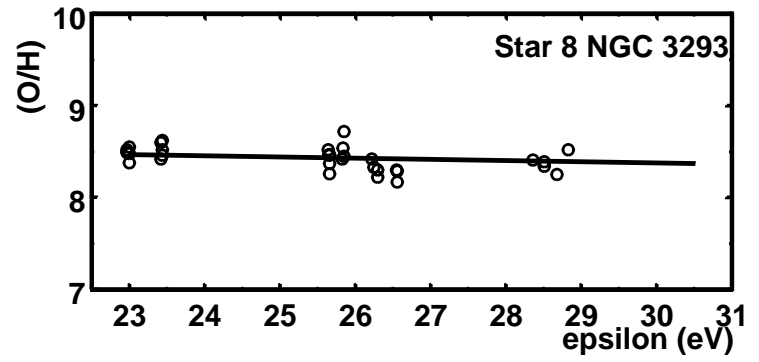

Fig. 4. Oxygen abundance from individual O II lines vs. their excitation potentials.

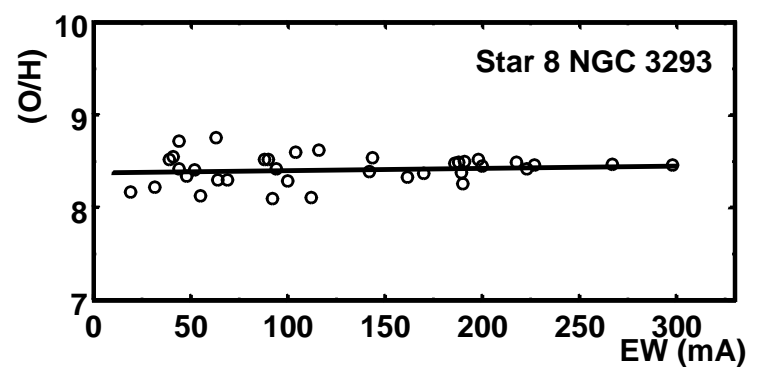

Fig. 5. Oxygen abundance from individual lines vs. their equivalent widths: determination of the microturbulent velocity.

consideration of the N II and O II lines are mutually consistent. In Fig. 5 we show an example of microturbulence velocity determination. A visible dependence between the abundance results from individual O II/ N II lines on their equivalent widths appear when the microturbulent velocity is varied within $10-15 \%$ of the adopted value.

Results of the NLTE $\xi_{\mathrm{t}}$ determinations are given in Table 2. As one can see, for some stars, the derived microturbulent velocities are rather high. Let us briefly discuss the high values found for B supergiants and $\mathrm{O}$ stars in the sample.

In several studies, it was found that some additional significant nonthermal broadening is required to explain observed line widths in the spectra of early B supergiant stars. For example, values in the range of $20-35 \mathrm{~km} \mathrm{~s}^{-1}$ were reported by Lamers (1972), Van Helden (1972), Lennon \& Dufton (1986), Gies \& Lambert (1992), Smartt et al. (1997), McErlean et al. (1998), Vrancken et al. (2000). Such velocities are of the order of magnitude of, or higher than, the thermal velocity of the hydrogen atoms in the atmospheres of these stars. For main-sequence early B stars, high microturbulent velocities were also derived by e.g. Gies \& Lambert (1992).

It is known that the atmospheric structure of hot and luminous stars is significantly complicated by the presence of large stellar winds. These winds are characterized by strong high-velocity turbulence (shock-wave perturbed winds). As shown by Groenewegen \& Lamers (1989), such a turbulence in A-B-O supergiant stars and $\mathrm{O}$ dwarfs can be described by a $\xi_{\text {turb }}$ parameter which equals approximately $10 \%$ of the wind terminal velocity. On average, O stars (both main-sequence and supergiants) have higher terminal wind velocities, therefore a stronger turbulence can be expected for them (even for A supergiants velocities of about $20 \mathrm{~km} \mathrm{~s}^{-1}$ are found; see McCarthy et al. 1997). As wind turbulence and a significant velocity gradient in the atmosphere (caused by the wind) can, to some extent, be imprinted on the profiles of the strong photospheric lines in the spectra of hot luminous stars, rather high microturbulent parameters are derived (see Kudritzki 1992; Lamers \& Achmad 1994; and discussion in McErlean et al. 1998).

Admittedly, one could artificially decrease the microturbulent velocities of the programme stars by excluding the strong lines (say, with equivalent widths of about $300 \mathrm{~m} \AA$ ). Nevertheless, this is not justified since these lines are appropriate for NLTE analysis: thus they are included in the present study. However, in agreement with Dufton (1972) and Smartt et al. (1997), we do not attach a great physical meaning to the determined microturbulent velocities, and we consider the $\xi_{\mathrm{t}}$ value as an empirical fitting parameter to avoid any systematic trend in the $[\mathrm{O} / \mathrm{H}]$ or $[\mathrm{N} / \mathrm{H}]$ vs. equivalent width relations.

\subsection{Projected rotational velocities}

In order to estimate the projected rotational velocities for the programme stars we used the SYNSPEC code (Hubeny et al. 1994). Determination of $v \sin i$ values in the studied stars was carried out by fitting the synthetic and observed profiles of the line He I $4437 \AA$. This line was used because:

1. it is a single, unblended line;

2. it is not very strong in any of the stars, and its profile is not expected to be sensitive to NLTE effects;

3 . it is also quite insensitive to the microturbulent velocity (see, e.g., McErlean et al. 1998).

For all the stars the fit was performed using the code SYNSPEC. In Fig. 6 we show some examples of the profile fitting. Estimated projected rotational velocities are presented in Table 2. The estimated error in the $v \sin i$ determinations is about $20 \%$ of the adopted value. Recently, Garcia \& Mermilliod (2001) presented projected rotational velocities for stars in NGC 6231. Our results for the stars of this study are in marginal agreement with their determinations, but in average slightly smaller.

\section{Elemental abundances}

Elemental abundances were determined using all available CNO lines with equivalent widths up to $300 \mathrm{~m} \AA$. The results are presented in Table 3. Some examples of observed and NLTE synthetic profiles for selected CNO lines are shown in Figs. 7-9. A comparison between NLTE and LTE abundances (which were also calculated, but not presented in this paper), shows that for the majority of the programme stars the differences between NLTE and LTE are typically about $0.1-0.2 \mathrm{dex}$, although in a few cases these differences exceed 0.5 dex.

Helium abundances were determined from the line He I $4437 \AA$. This absorption line is not strong in any 
Table 3. NLTE abundances of CNO elements and LTE abundance of He.

\begin{tabular}{|c|c|c|c|c|c|c|c|c|}
\hline Star & $(\mathrm{C} / \mathrm{H})$ & $\sigma$ & $(\mathrm{N} / \mathrm{H})$ & $\sigma$ & $(\mathrm{O} / \mathrm{H})$ & $\sigma$ & $(\mathrm{He} / \mathrm{H})$ & $\sigma$ \\
\hline \multicolumn{9}{|c|}{ NGC 3293} \\
\hline 8 & 8.22 & 0.11 & 7.78 & 0.15 & 8.41 & 0.15 & 11.20 & \\
\hline 13 & 8.38 & 0.10 & 7.81 & 0.13 & 8.65 & 0.15 & 11.12 & \\
\hline 16 & 8.10 & 0.14 & 7.71 & 0.16 & 8.42 & 0.15 & 11.13 & \\
\hline 18 & 8.16 & 0.15 & 7.69 & 0.16 & 8.65 & 0.15 & 11.07 & \\
\hline 20 & 8.15 & - & 7.92 & 0.12 & 8.52 & 0.17 & 11.25 & \\
\hline 22 & 8.18 & - & 7.68 & 0.13 & 8.34 & 0.15 & 11.25 & \\
\hline Mean & 8.20 & 0.10 & 7.77 & 0.10 & 8.50 & 0.13 & 11.15 & 0.07 \\
\hline \multicolumn{9}{|c|}{ NGC 4755} \\
\hline 2 & 7.84 & 0.07 & 7.90 & 0.17 & 8.10 & 0.12 & 11.40 & \\
\hline 3 & 7.70 & 0.02 & 7.97 & 0.12 & 8.14 & 0.16 & 11.25 & \\
\hline 5 & 8.30 & - & 7.94 & 0.05 & 8.36 & 0.18 & 11.15 & \\
\hline 6 & 8.65 & - & 8.04 & 0.32 & 8.60 & 0.16 & 11.15 & \\
\hline 7 & 8.11 & 0.08 & 8.13 & 0.19 & 8.42 & 0.28 & 11.15 & \\
\hline 18 & 8.01 & 0.18 & 7.46 & 0.15 & 8.30 & 0.25 & 10.95 & \\
\hline 106 & 8.00 & 0.13 & 8.09 & 0.16 & 8.12 & 0.18 & 11.30 & \\
\hline 414 & 7.95 & - & 7.83 & 0.15 & 8.86 & 0.17 & 11.10 & \\
\hline Mean & 8.07 & 0.29 & 7.92 & 0.21 & 8.36 & 0.26 & 11.18 & 0.14 \\
\hline \multicolumn{9}{|c|}{ NGC 6231} \\
\hline 161 & 8.30 & 0.15 & 7.95 & - & 7.76 & 0.13 & 10.50 & \\
\hline 232 & 8.48 & 0.17 & 7.98 & 0.29 & 8.63 & 0.18 & 11.00 & \\
\hline 286 & 8.43 & 0.17 & 7.79 & 0.30 & 8.30 & 0.21 & 10.80 & \\
\hline 287 & 8.13 & 0.25 & 7.75 & 0.17 & 8.92 & 0.16 & 10.73 & \\
\hline 292 & 8.09 & - & 7.80 & - & 8.48 & 0.15 & 11.03 & \\
\hline 293 & - & - & - & - & 8.20 & 0.04 & 10.63 & \\
\hline 323 & - & - & - & - & 7.81 & 0.02 & 10.95 & \\
\hline Mean & 8.29 & 0.17 & 7.85 & 0.10 & 8.30 & 0.42 & 10.81 & 0.20 \\
\hline $\operatorname{Sun}^{a}$ & 8.55 & & 7.97 & & 8.87 & & 10.99 & \\
\hline
\end{tabular}

${ }^{a}$ Solar abundances are from Grevesse et al. (1996).

of the programme stars. It is not seen in the spectrum of NGC 6231-323, where two other helium lines were used: $4009 \AA$ and $4143 \AA$.

One may question if LTE analysis of helium lines yields reliable abundances of this element. This question has been addressed in the literature. The most comprehensive NLTE studies of helium lines are those by Auer \& Mihalas (1973) and Dimitrov \& Kubat (1988). In both studies, the authors investigated departures from LTE (specifically the behaviour of " $b$ " factors) for helium lines in a wide spectral region. They concluded that over a wide range of effective temperatures and gravities, NLTE corrections are negligible for the blue-violet spectral region, but that they become very significant in the red-infrared. In particular, for the line He I $4437 \AA$, the difference between NLTE and LTE equivalent widths reaches only $5 \%$. Thus, it is comparable with the error of our equivalent width measurements, and it cannot significantly affect the helium abundances that we infer. Accordingly, the latter appear reliable.

\section{Uncertainties}

As mentioned above, the photometric indices for a given star reported by different authors can, in some cases, differ rather markedly. This may be partly caused by differences in detector sensitivity (see remarks in Balona 1994). But most likely, actual stellar variability is a significant contribution. As a matter of fact, almost all our programme stars are known to be variable (for example, stars 16, 18, 20 in NGC 3293, stars 2, 6, 7, 18, 106 in NGC 4755, stars $161,232,282,292,293$ in NGC 6231). Even if a star is not reported as a variable, its photometric variability can be suspected from the existence of rather large radial velocity variations (SIMBAD database).

The influence of errors in the adopted atmospheric parameters on the derived abundances is illustrated for the 


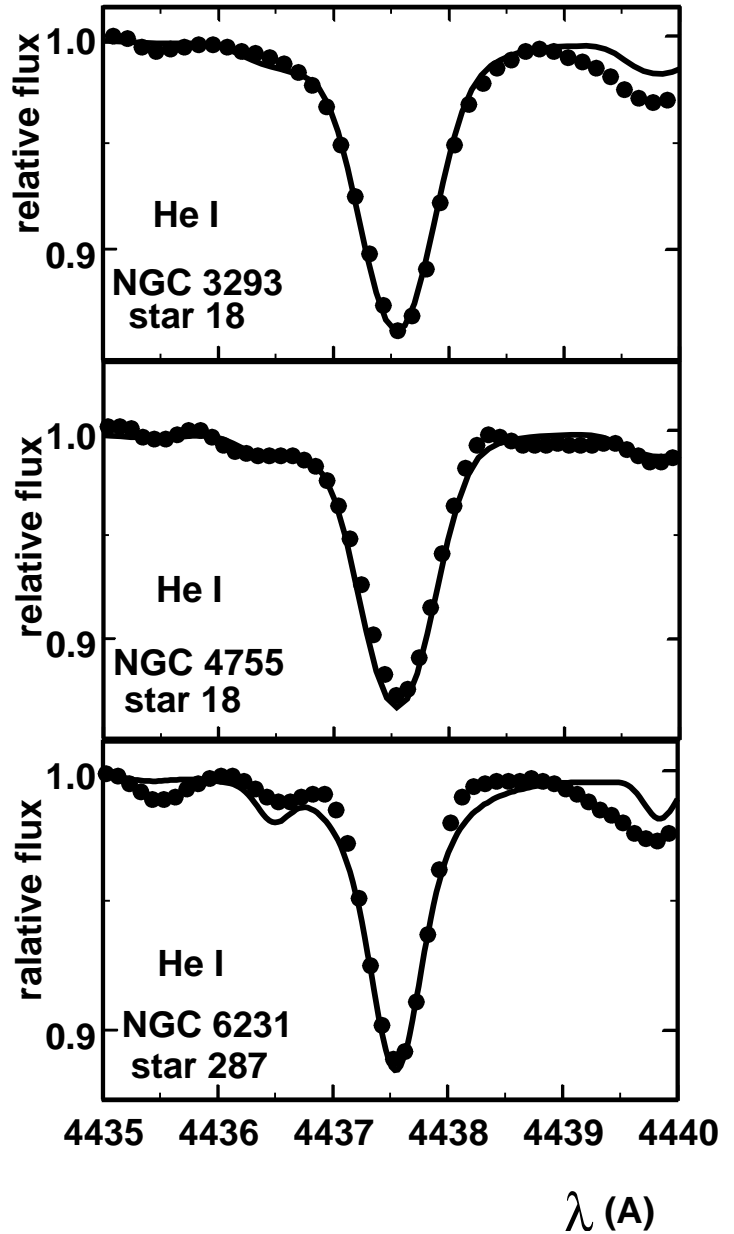

Fig. 6. Observed and calculated profiles of the line He I $4437 \AA$ for some programme stars.

case of star NGC 3293-16. Based on published photometric measurements of this star, we obtain: $(b-y)=0.045 \pm$ $0.012, m_{1}=0.047 \pm 0.022, c_{1}=0.015 \pm 0.016$, and $\beta=2.592 \pm 0.002$. In the simplest case (all the indices are either simultaneously increased, or simultaneously decreased), we derive the following parameter uncertainties: $T_{\text {eff }} \approx 24400 \mathrm{~K}-25800 \mathrm{~K}, \log g \approx 3.7-3.8$. With both the temperature and the gravity at the lower limit of their uncertainty range, the derived NLTE abundances are:

$(\mathrm{C} / \mathrm{H})=8.06 \pm 0.16$,

$(\mathrm{N} / \mathrm{H})=7.67 \pm 0.16$

$(\mathrm{O} / \mathrm{H})=8.44 \pm 0.14$.

With the highest temperature and highest gravity, the abundances are:

$(\mathrm{C} / \mathrm{H})=8.15 \pm 0.13$

$(\mathrm{N} / \mathrm{H})=7.77 \pm 0.17$

$(\mathrm{O} / \mathrm{H})=8.40 \pm 0.15$.

Note that with the adopted parameters for this star $\left(T_{\text {eff }}=25000 \mathrm{~K}, \log g=3.75\right)$, we get:

$(\mathrm{C} / \mathrm{H})=8.10 \pm 0.15$,

$(\mathrm{N} / \mathrm{H})=7.71 \pm 0.16$

$(\mathrm{O} / \mathrm{H})=8.42 \pm 0.15$.
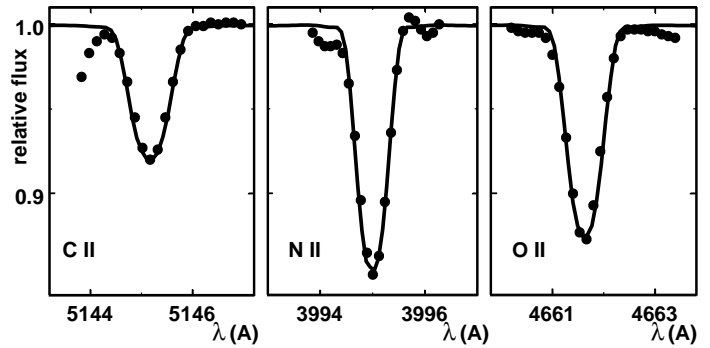

Fig. 7. Observed and calculated profiles of selected CNO lines for star NGC 3293-13.
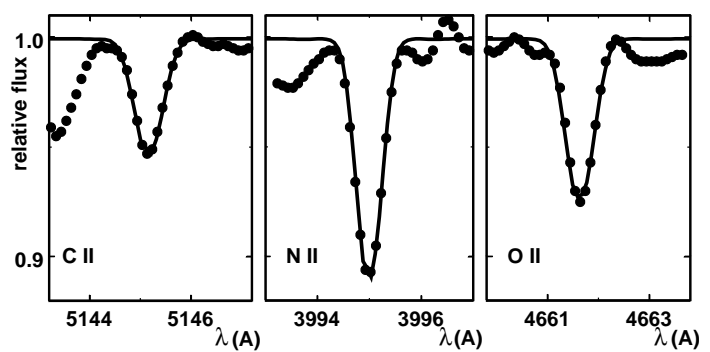

Fig. 8. Same as Fig. 7 but for star NGC 4755-18.

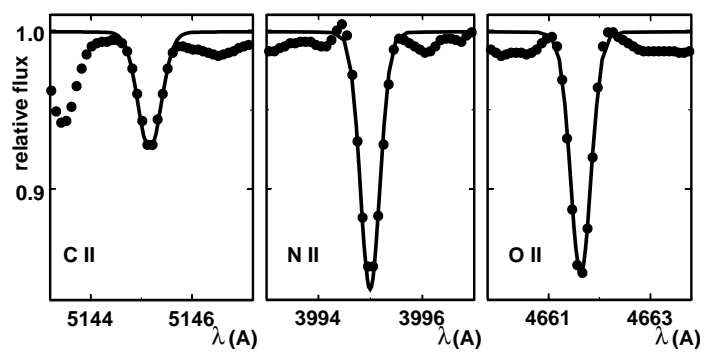

Fig. 9. Same as Fig. 7 but for star NGC 6231-287.

For some of the studied stars, another important source of uncertainty comes from the fact that they belong to double-lined binary systems (see Sect. 4). In such systems, the observed spectrum results from the superposition of the individual spectra of the two components. Our observational material does not allow us to separate the contributions of each of them. If the lines of the components could be distinguished, their equivalent widths would be reduced due to the dilution of the continuum of one star by that of the other. In the present case, the lines appear single in all the stars observed, so that they must be contributed by both members of the binary. Accordingly, our analysis is self-consistent, in that the abundances are derived from the same combination of the contributions of the two components as the stellar atmospheric parameters. The "average" abundances that are determined in that way can be expected to represent reasonably well the composition of the two stars, provided that they are sufficiently similar, in particular in mass and evolutionary state. This seems rather likely for members of such early type SB2s - any significant mass difference between the two members of a pair would almost unavoidably imply such a difference of luminosity that the less massive one 


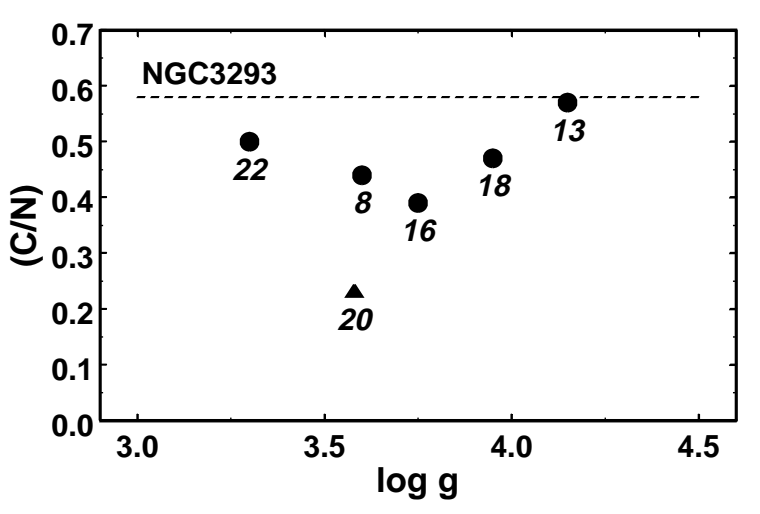

Fig. 10. (C/N) ratio vs. surface gravity for the stars of NGC 3293. Circles identify the stars with projected rotational velocity less than $55 \mathrm{~km} \mathrm{~s}^{-1}$, triangles represent the stars with $v \sin i>95 \mathrm{~km} \mathrm{~s}^{-1}$. The solar $(\mathrm{C} / \mathrm{N})$ ratio is shown by the dashed line.

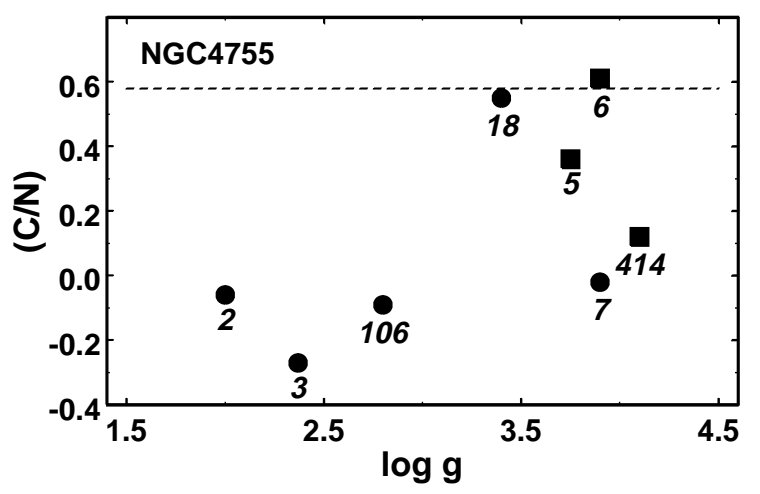

Fig. 11. (C/N) ratio vs. surface gravity for the stars of NGC 4755. Squares correspond to the stars with projected rotational velocity between $55 \mathrm{~km} \mathrm{~s}^{-1}$ and $95 \mathrm{~km} \mathrm{~s}^{-1}$.

would not contribute significantly to the observed colours and spectrum. In other words, although they cannot be exactly quantified from the analyzed spectra, uncertainties of abundances determinations in the SB2 systems of the sample are expected to be small. However, their possible influence should be kept in mind in the interpretation of the results.

\section{Discussion}

\subsection{Helium abundance}

Ahumada \& Lapasset (1995) give the ages of NGC 3293 $(\log A=7.40)$, NGC $4755(\log A=6.85)$, and NGC 6231 $(\log A=6.50)$. From Table 3 one can infer a mean helium abundance for NGC 3293 of $(\mathrm{He} / \mathrm{H})=11.15 \pm 0.07$; for NGC 4755, it is $(\mathrm{He} / \mathrm{H})=11.18 \pm 0.14$, and for NGC 6231, $(\mathrm{He} / \mathrm{H})=10.81 \pm 0.20$. The surface abundance of helium in the youngest of the three studied clusters, NGC 6231, may be marginally less than in the two older ones. Nevertheless, the difference is too small to derive any evolutionary conclusion. The supergiants of our sample do not differ significantly in their helium abundance from other programme stars, but they may show a hint of helium enhancement.

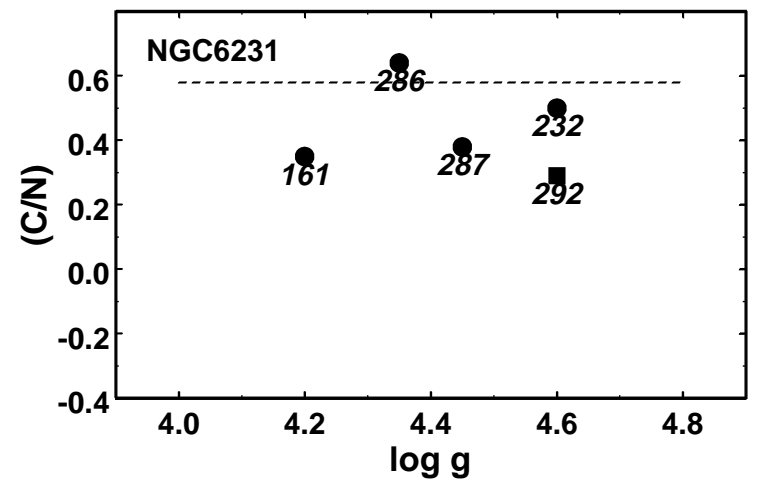

Fig. 12. (C/N) ratio vs. surface gravity for the stars of NGC 6231.

\subsection{Carbon, nitrogen, and mixing}

In Figs. 10 to 12, the ratio $(\mathrm{C} / \mathrm{N})$ is plotted against the surface gravity of the sample stars. If B-type mainsequence stars undergo large-scale internal mixing, the consequences should show through in the ratio of the surface abundances of carbon to nitrogen. At first sight, Figs. 10 to 12 may appear to support the view that CNcycled material can appear at the surface of some B stars during their main-sequence lifetime. For instance, this is suggested by the scatter of the $(\mathrm{C} / \mathrm{N})$ values among the stars of NGC 6231, which all have large surface gravities. Nevertheless, closer inspection of Table 3 reveals that the main contribution to low $(\mathrm{C} / \mathrm{N})$ values comes from lower carbon abundances, while nitrogen remains nearly solar in almost all cases. Even for the two stars of NGC 4755 with the lowest gravities (stars 2 and 3), where mixing processes might be expected to modify the surface abundances, nitrogen appears to be solar. One possible interpretation of this puzzling result is to suppose that in some stars, present-day $\mathrm{C}$ and $\mathrm{N}$ surface abundances are really altered by CNO-cycle processing, but that the initial abundances of these elements were subsolar.

The problem of subsolar metallicities in B stars has been widely debated recently. This feature of the chemical composition of B stars has been found in virtually all studies (see, e.g., Gies \& Lambert 1992; Cunha \& Lambert 1994; Daflon et al. 1999; Andrievsky et al. 1999; Rolleston et al. 2000). Subsolar abundances were reported for C-N-O elements, as well as for heavier species. Hou et al. (2000) conclude that at present there is no satisfactory explanation for the supermetallicity of the Sun relative to young objects (specifically B stars) in the solar neighbourhood. Luck et al. (2000) proposed that conclusions about subsolar metallicities may suffer from ignoring effects such as atomic diffusion in atmospheres of $\mathrm{B}$ main-sequence stars. Another explanation was recently proposed by Snow (2000). Such processes as sedimentation and ambipolar diffusion during stellar formation could lead to some decrease in the abundances of some elements.

Possibly, the long-standing problem of subsolar $\mathrm{C}$ and $\mathrm{N}$ abundances in B stars may eventually find an even simpler solution, as recently happened with oxygen after 
revision of its abundance in the Sun. A recent work of Holweger (2001) based on optical and infra-red lines placed the solar oxygen abundance at $(\mathrm{O} / \mathrm{H})=8.74 \pm$ 0.08 , that is, significantly lower than accepted before. An even lower oxygen abundance in the Sun was recently deduced by Prieto et al. (2001). By applying a 3-D timedependent hydrodynamical model of the solar atmosphere, those authors re-analyzed the forbidden line [O I] $6300 \AA$, finding that the oxygen abundance is $8.69 \pm 0.05$. In fact, the mean oxygen abundances that we derive in the studied clusters are now, within errors, in marginal agreement with the latter value.

As one can see from Figs. 10 to 12, the mainsequence stars (i.e., those having gravities higher than, say, $3.75 \mathrm{dex}$ ) are mostly near the line corresponding to atmospheric $\mathrm{C}-\mathrm{N}$ abundances that have not yet been altered through mixing with $\mathrm{CN}$-processed material (stars 7 and 414 in NGC 4755 are two obvious exceptions). Among the group of stars with $\log g>3.75$, there are stars with nearly solar abundances of carbon and nitrogen, as well as stars with decreased abundances of both carbon and nitrogen. If the net result of the competing effects of upward radiative forces and downward gravitational settling is similar for carbon and nitrogen ions, selective radiative diffusion could, in principle, lead to a comparable dilution of $\mathrm{C}$ and $\mathrm{N}$ in the atmospheres of $\mathrm{B}$ stars. This process can be effective only if the rotational velocity is not too high (but an upper limit has not been estimated for B stars). For example, star NGC 4755-6, with $v \sin i=95 \mathrm{~km} \mathrm{~s}^{-1}$, has solar $\mathrm{C}$ and $\mathrm{N}$ abundances, while star NGC 3293-22, with a more moderate rotational velocity $\left(v \sin i=55 \mathrm{~km} \mathrm{~s}^{-1}\right)$ shows both decreased carbon and nitrogen. The latter case may indicate that rotational velocities as high as $50-100 \mathrm{~km} \mathrm{~s}^{-1}$ do not effectively prevent radiative diffusion in the atmosphere.

Several stellar evolutionary grids have been computed with particular attention to the evolution of the surface abundances (e.g., Maeder \& Meynet 1988; Schaller et al. 1992). In these tables, in the models with masses $9-12 M_{\odot}$ (which correspond to the main-sequence stars of the present sample), one can note that the first change of the surface abundances of carbon and nitrogen happens when the model reaches the red giant region. Contrary to this prediction of standard stellar evolution, in this study, CNprocessed material seems to appear at the stellar surface for hot supergiants (stars 2, 3, 106 in NGC 4755). These stars could have already passed the red giant stage. A possibility to create a N-rich main-sequence star through rotationally induced mixing was considered by Maeder (1987). The author succeeded in showing that this effect can modify the surface abundances of a $25 M_{\odot}$ rapidly rotating star. Whether this should be the case for hot supergiant stars with lower masses (and rather small projected rotation velocities) is not clear.

Very recently Heger \& Langer (2000) performed a theoretical study considering the evolution of surface abundances for rotating massive stars. The authors found that the abundance ratios $(\mathrm{C} / \mathrm{N}),(\mathrm{C} / \mathrm{O})$ and $(\mathrm{N} / \mathrm{O})$ significantly depend upon the rotational velocity. For example, after approximately $10 \mathrm{Myr}$ (the mean age of our sample of cluster) surface $[\mathrm{C} / \mathrm{N}]$ ratio should be about -0.3 dex if the star rotates with equatorial velocity $200 \mathrm{~km} \mathrm{~s}^{-1}$. From Figs. 10-12 one can see that the great majority of our non-supergiant stars show a similar value of $(\mathrm{C} / \mathrm{N})$, but we should note that they seem to rotate slowly for their spectral types (although some of them can be pole-on rotators), and for rotational velocities of about $100 \mathrm{~km} \mathrm{~s}^{-1}$ a negligible modification on the surface CNO abundances is expected (see Heger \& Langer's Fig. 9). Also, according to Howarth \& Smith (2001) the only very rapid rotators are nitrogen enhanced.

On the other hand, Heger \& Langer (2000) predict that rotation should decrease surface $(\mathrm{C} / \mathrm{O})$ ratio. In fact, the typical $(\mathrm{C} / \mathrm{O})$ value for our program stars is about the same as for the Sun (but with significant deviations, both positive and negative, for some stars), therefore the comparison between our determinations and the theoretical predictions does not seem to support the supposition that surface abundances in massive young B stars are rotationally altered.

\section{Conclusion}

The C-N abundances determined for the sample of stars considered in this study do not show clear evidence for the occurrence of internal mixing for most main-sequence stars. Only three non-supergiant stars, NGC 4755-7, NGC 4755-414, and NGC 3293-20 seem to show an enhanced nitrogen abundance together with decreased carbon. Two of them do not have a large projected rotational velocity (but admittedly, they might be rapid rotators seen almost pole-on). In the lower gravity stars (stars 2, 3 and 106 in NGC 4755), CNO-processed material appears to be present at the surface, suggesting that some kind of mixing has occurred. This conclusion is based on the assumption that initial abundances of carbon and nitrogen in these stars were subsolar. The problem of subsolar abundances itself is not yet resolved and deserved further attention. If the hypothesis of initial subsolar abundances is not valid, then one needs to identify a mechanism causing an appreciable carbon depletion in B stars (supergiants, in particular), while nitrogen remains nearly unchanged.

Acknowledgements. SMA would like to express his gratitude to FAPESP for the visiting professor fellowship (No. 2000/065873) and to Instituto Astronômico e Geofísico, Universidade de São Paulo (Brazil) for providing facility support during a productive stay in Brazil. The authors are indebted to the referee Dr. D. R. Gies for the very detailed and constructive review of the paper. The SIMBAD database was used.

\section{Appendix A: Equivalent width measurements}

Tables A.1, A.2 and A.3 give the equivalent widths of the spectral lines measured in the spectra of the programme stars of, resp., NGC 3293, NGC 4755, and NGC 6231. 
Table A.1. Equivalent widths of the lines in spectra of stars of NGC 3293.

\begin{tabular}{|c|c|c|c|}
\hline & & & \\
\hline Wave & code & $\log g f$ & $E_{\text {low }}, \mathrm{eV}$ \\
\hline 4437.55 & 2.00 & -2.03 & 21.217 \\
\hline 3920.68 & 6.01 & -0.21 & 16.332 \\
\hline 4074.54 & 6.01 & 0.39 & 24.369 \\
\hline 4374.28 & 6.01 & 0.66 & 24.652 \\
\hline 5139.17 & 6.01 & -0.74 & 20.703 \\
\hline 5143.49 & 6.01 & -0.24 & 20.703 \\
\hline 5145.16 & 6.01 & 0.16 & 20.709 \\
\hline 5151.09 & 6.01 & -0.20 & 20.709 \\
\hline 3955.85 & 7.01 & -0.78 & 18.465 \\
\hline 3995.00 & 7.01 & 0.28 & 18.496 \\
\hline 4035.08 & 7.01 & 0.62 & 23.123 \\
\hline 4041.31 & 7.01 & 0.85 & 23.141 \\
\hline 4043.53 & 7.01 & 0.74 & 23.131 \\
\hline 4082.27 & 7.01 & 0.15 & 23.131 \\
\hline 4171.59 & 7.01 & 0.28 & 23.195 \\
\hline 4176.16 & 7.01 & 0.60 & 23.195 \\
\hline 4199.98 & 7.01 & 0.03 & 23.245 \\
\hline 4227.74 & 7.01 & -0.07 & 21.598 \\
\hline 4237.05 & 7.01 & 0.55 & 23.241 \\
\hline 4241.79 & 7.01 & 0.71 & 23.245 \\
\hline 4432.74 & 7.01 & 0.58 & 23.414 \\
\hline 4442.02 & 7.01 & 0.31 & 23.420 \\
\hline 4447.03 & 7.01 & 0.29 & 20.408 \\
\hline 4530.41 & 7.01 & 0.67 & 23.473 \\
\hline 4607.15 & 7.01 & -0.49 & 18.461 \\
\hline 4613.87 & 7.01 & -0.73 & 18.465 \\
\hline 4621.39 & 7.01 & -0.54 & 18.465 \\
\hline 4630.54 & 7.01 & 0.13 & 18.482 \\
\hline 4643.09 & 7.01 & -0.34 & 18.482 \\
\hline 4788.14 & 7.01 & -0.38 & 20.652 \\
\hline 4803.29 & 7.01 & -0.12 & 20.664 \\
\hline 5002.70 & 7.01 & -1.02 & 18.461 \\
\hline 5005.15 & 7.01 & 0.61 & 20.664 \\
\hline 5007.33 & 7.01 & 0.16 & 20.939 \\
\hline 5010.62 & 7.01 & -0.52 & 18.465 \\
\hline 5025.66 & 7.01 & -0.44 & 20.664 \\
\hline 5045.10 & 7.01 & -0.33 & 18.482 \\
\hline 3945.04 & 8.01 & -0.69 & 23.418 \\
\hline 3982.72 & 8.01 & -0.67 & 23.440 \\
\hline 4048.24 & 8.01 & -0.37 & 28.692 \\
\hline 4062.91 & 8.01 & -0.09 & 28.704 \\
\hline 4071.24 & 8.01 & -0.09 & 28.692 \\
\hline 4072.16 & 8.01 & 0.53 & 25.648 \\
\hline 4075.87 & 8.01 & 0.69 & 25.664 \\
\hline 4078.86 & 8.01 & -0.26 & 25.637 \\
\hline 4083.93 & 8.01 & 0.15 & 28.682 \\
\hline 4085.12 & 8.01 & -0.14 & 25.648 \\
\hline 4087.20 & 8.01 & 0.53 & 28.675 \\
\hline 4092.93 & 8.01 & -0.25 & 25.664 \\
\hline 4110.78 & 8.01 & -0.89 & 25.836 \\
\hline 4112.03 & 8.01 & -0.78 & 25.847 \\
\hline 4119.22 & 8.01 & 0.48 & 25.847 \\
\hline 4129.31 & 8.01 & -1.12 & 25.836 \\
\hline 4132.79 & 8.01 & -0.07 & 25.830 \\
\hline 4153.28 & 8.01 & 0.08 & 25.836 \\
\hline 4156.52 & 8.01 & -0.79 & 25.847 \\
\hline 4185.44 & 8.01 & 0.71 & 28.356 \\
\hline 4189.79 & 8.01 & 0.80 & 28.359 \\
\hline 4275.53 & 8.01 & 0.76 & 28.856 \\
\hline 4291.27 & 8.01 & -0.23 & 28.820 \\
\hline 4294.87 & 8.01 & 0.36 & 28.829 \\
\hline 4307.34 & 8.01 & -0.05 & 28.837 \\
\hline 4317.15 & 8.01 & -0.32 & 22.965 \\
\hline 4319.64 & 8.01 & -0.32 & 22.978 \\
\hline 4325.76 & 8.01 & -1.06 & 22.965 \\
\hline 4366.91 & 8.01 & -0.24 & 22.998 \\
\hline 4369.31 & 8.01 & -0.35 & 26.224 \\
\hline 4414.88 & 8.01 & 0.17 & 23.440 \\
\hline 4416.97 & 8.01 & 0.04 & 23.418 \\
\hline 4443.04 & 8.01 & 0.00 & 28.356 \\
\hline 4448.34 & 8.01 & 0.13 & 28.359 \\
\hline 4452.38 & 8.01 & -0.74 & 23.440 \\
\hline 4465.44 & 8.01 & 0.34 & 30.423 \\
\hline 4591.01 & 8.01 & 0.45 & 25.660 \\
\hline 4596.20 & 8.01 & 0.29 & 25.660 \\
\hline 4609.37 & 8.01 & 0.67 & 29.067 \\
\hline 4610.17 & 8.01 & -0.17 & 29.061 \\
\hline 4638.86 & 8.01 & -0.30 & 22.965 \\
\hline 4649.14 & 8.01 & 0.31 & 22.998 \\
\hline 4661.64 & 8.01 & -0.17 & 22.978 \\
\hline 4673.75 & 8.01 & -1.07 & 22.978 \\
\hline 4676.24 & 8.01 & -0.30 & 22.998 \\
\hline 4696.36 & 8.01 & -1.31 & 22.998 \\
\hline 4699.19 & 8.01 & 0.37 & 28.508 \\
\hline 4701.21 & 8.01 & 0.07 & 28.828 \\
\hline 4703.26 & 8.01 & 0.21 & 28.511 \\
\hline 4705.32 & 8.01 & 0.57 & 26.248 \\
\hline 4709.99 & 8.01 & -0.47 & 26.224 \\
\hline 4890.85 & 8.01 & -0.33 & 26.303 \\
\hline 4906.82 & 8.01 & -0.03 & 26.303 \\
\hline 4941.11 & 8.01 & 0.08 & 26.552 \\
\hline 4943.00 & 8.01 & 0.37 & 26.560 \\
\hline 4955.74 & 8.01 & -0.42 & 26.560 \\
\hline
\end{tabular}


Table A.2. Equivalent widths of the lines in spectra of stars of NGC 4475.

\begin{tabular}{|c|c|c|c|c|c|c|c|c|c|c|c|}
\hline & & & & \multicolumn{8}{|c|}{$E W, \mathrm{~m} \AA$} \\
\hline Wave & code & $\log g f$ & $E_{\text {low }}, \mathrm{eV}$ & 2 & 3 & 5 & 6 & 7 & 18 & 106 & 414 \\
\hline 4437.55 & 2.00 & -2.03 & 21.217 & 112.0 & 133.0 & 114.0 & 117.0 & 115.3 & 104.0 & 104.0 & 134.6 \\
\hline 3918.97 & 6.01 & -0.51 & 16.331 & & & & & & 100.0 & & \\
\hline 3920.68 & 6.01 & -0.21 & 16.332 & 184.5 & 199.0 & & & 117.0 & 90.5 & 155.0 & 102.0 \\
\hline 4374.28 & 6.01 & 0.66 & 24.652 & & & & & 29.3 & & & \\
\hline 5122.09 & 6.01 & -0.53 & 20.844 & & 24.3 & & & & 24.5 & & \\
\hline 5143.49 & 6.01 & -0.24 & 20.703 & & & & & & 32.0 & & \\
\hline 5145.16 & 6.01 & 0.16 & 20.709 & & & & & 43.0 & 36.3 & 64.0 & \\
\hline 5151.09 & 6.01 & -0.20 & 20.709 & & & & 70.0 & & 26.0 & & \\
\hline 3955.85 & 7.01 & -0.78 & 18.465 & 75.0 & 76.0 & & & 79.0 & & & \\
\hline 3995.00 & 7.01 & 0.28 & 18.496 & 212.0 & 241.0 & 126.9 & 133.0 & & 69.6 & 294.0 & \\
\hline 4035.08 & 7.01 & 0.62 & 23.123 & 41.8 & 111.0 & 79.0 & 102.0 & 75.0 & 45.0 & 89.0 & \\
\hline 4041.31 & 7.01 & 0.85 & 23.141 & 81.1 & & & & 69.7 & 32.8 & 114.0 & 50.4 \\
\hline 4043.53 & 7.01 & 0.74 & 23.131 & 52.0 & & & & & & 61.0 & \\
\hline 4082.27 & 7.01 & 0.15 & 23.131 & & & & & & & 20.4 & \\
\hline 4171.59 & 7.01 & 0.28 & 23.195 & & 18.6 & & & & & & \\
\hline 4199.98 & 7.01 & 0.03 & 23.245 & 19.6 & & & & & & & \\
\hline 4227.74 & 7.01 & -0.07 & 21.598 & & 37.0 & & & & & 58.0 & \\
\hline 4237.05 & 7.01 & 0.55 & 23.241 & & 68.0 & & 66.5 & 78.0 & 31.0 & & \\
\hline 4241.79 & 7.01 & 0.71 & 23.245 & & 102.0 & & & & 39.0 & & \\
\hline 4432.74 & 7.01 & 0.58 & 23.414 & & & & & & 12.5 & & \\
\hline 4447.03 & 7.01 & 0.29 & 20.408 & 96.5 & 103.0 & & & 112.0 & 33.5 & 143.0 & \\
\hline 4530.41 & 7.01 & 0.67 & 23.473 & & 74.0 & & & 63.0 & 32.0 & & \\
\hline 4601.48 & 7.01 & -0.37 & 18.465 & & 120.0 & & & 123.0 & 40.0 & 150.0 & \\
\hline 4607.15 & 7.01 & -0.49 & 18.461 & & 121.5 & & & 79.5 & 30.3 & 149.0 & 33.6 \\
\hline 4613.87 & 7.01 & -0.73 & 18.465 & 74.0 & 86.5 & & & 93.0 & 31.0 & 129.0 & \\
\hline 4621.39 & 7.01 & -0.54 & 18.465 & 54.0 & 82.5 & & & 86.0 & 42.0 & 124.0 & \\
\hline 4630.54 & 7.01 & 0.13 & 18.482 & 199.0 & 240.1 & 201.0 & 140.0 & 126.0 & 57.0 & 318.0 & 67.2 \\
\hline 4643.09 & 7.01 & -0.34 & 18.482 & 123.0 & 158.5 & & & 91.0 & 36.4 & & \\
\hline 4779.72 & 7.01 & -0.56 & 20.645 & & & & 50.0 & 30.0 & & 62.0 & \\
\hline 4788.14 & 7.01 & -0.38 & 20.652 & 52.7 & 46.4 & & & 38.7 & 12.4 & & \\
\hline 4803.29 & 7.01 & -0.12 & 20.664 & & 86.0 & & & & & 100.0 & \\
\hline 5005.15 & 7.01 & 0.61 & 20.664 & 120.0 & 155.0 & 150.0 & & & 46.0 & 217.0 & 75.0 \\
\hline 5007.33 & 7.01 & 0.16 & 20.939 & & 74.0 & & & 53.4 & 30.0 & 93.0 & \\
\hline 5010.62 & 7.01 & -0.52 & 18.465 & & 113.0 & & & & 24.0 & 142.0 & 35.0 \\
\hline 5045.10 & 7.01 & -0.33 & 18.482 & 110.0 & 141.0 & & & 85.0 & 30.0 & 196.0 & \\
\hline 3945.04 & 8.01 & -0.69 & 23.418 & & & 162.0 & & 73.5 & 50.0 & 93.0 & \\
\hline 3982.72 & 8.01 & -0.67 & 23.440 & 40.0 & & & & & 27.5 & 94.0 & \\
\hline 4072.16 & 8.01 & 0.53 & 25.648 & 69.7 & 82.0 & & & 100.0 & 44.0 & & \\
\hline 4075.87 & 8.01 & 0.69 & 25.664 & & & & & & 75.0 & & \\
\hline 4078.86 & 8.01 & -0.26 & 25.637 & & 26.1 & & & 65.0 & & 60.0 & \\
\hline 4087.20 & 8.01 & 0.53 & 28.675 & & & & & & 25.1 & 35.0 & \\
\hline 4092.93 & 8.01 & -0.25 & 25.664 & & & & & 64.0 & & 41.0 & \\
\hline 4119.22 & 8.01 & 0.48 & 25.847 & & 91.0 & & & 168.0 & 66.0 & 170.0 & \\
\hline 4132.79 & 8.01 & -0.07 & 25.830 & 34.0 & & & & & 32.0 & 92.0 & \\
\hline 4153.28 & 8.01 & 0.08 & 25.836 & 57.0 & 73.0 & 134.0 & 104.0 & & 47.4 & 101.5 & \\
\hline 4185.44 & 8.01 & 0.71 & 28.356 & 26.0 & 43.0 & 97.0 & 105.0 & 54.4 & 30.8 & 69.0 & 48.0 \\
\hline 4189.79 & 8.01 & 0.80 & 28.359 & 27.0 & & & 106.0 & 70.0 & 36.0 & 88.9 & \\
\hline 4317.15 & 8.01 & -0.32 & 22.965 & 72.0 & 91.0 & 207.0 & 160.0 & 104.4 & 53.0 & 173.0 & 78.0 \\
\hline 4319.64 & 8.01 & -0.32 & 22.978 & 74.0 & 97.0 & 192.0 & & 103.3 & 44.0 & 173.0 & 72.7 \\
\hline 4325.76 & 8.01 & -1.06 & 22.965 & 24.1 & 36.8 & & & 57.0 & 30.0 & 53.0 & \\
\hline 4366.91 & 8.01 & -0.24 & 22.998 & & 59.0 & 162.0 & & 99.0 & 49.0 & 183.0 & \\
\hline 4414.88 & 8.01 & 0.17 & 23.440 & 88.0 & 127.0 & & 213.0 & 136.0 & 61.5 & 257.0 & 77.7 \\
\hline 4416.97 & 8.01 & 0.04 & 23.418 & 86.0 & 111.0 & & & 128.0 & 57.8 & 194.0 & 74.2 \\
\hline 4452.38 & 8.01 & -0.74 & 23.440 & 46.3 & 37.1 & 74.0 & & 73.0 & 28.0 & 72.5 & \\
\hline 4591.01 & 8.01 & 0.45 & 25.660 & 70.0 & 75.0 & 179.0 & & 111.0 & 41.4 & 163.0 & 61.0 \\
\hline 4596.20 & 8.01 & 0.29 & 25.660 & & 62.0 & & & 104.0 & 50.0 & 128.7 & \\
\hline 4609.37 & 8.01 & 0.67 & 29.067 & & & & & 95.0 & 29.0 & & 46.3 \\
\hline 4638.86 & 8.01 & -0.30 & 22.965 & 78.0 & 87.5 & 235.0 & & 104.0 & 49.5 & 185.0 & 70.0 \\
\hline 4649.14 & 8.01 & 0.31 & 22.998 & 143.0 & 206.5 & & & 199.0 & 79.0 & & \\
\hline 4650.85 & 8.01 & -0.36 & 22.965 & & & & & & 55.0 & & \\
\hline 4661.64 & 8.01 & -0.17 & 22.978 & 94.0 & 94.0 & 210.0 & 133.0 & 102.0 & 48.9 & 209.0 & 71.0 \\
\hline 4673.75 & 8.01 & -1.07 & 22.978 & & & & & & 13.8 & 48.0 & \\
\hline 4676.24 & 8.01 & -0.30 & 22.998 & & & 217.0 & 143.0 & 105.0 & 31.9 & 164.0 & \\
\hline 4696.36 & 8.01 & -1.31 & 22.998 & & & & & 41.0 & & & \\
\hline 4699.19 & 8.01 & 0.37 & 28.508 & 32.4 & & 97.0 & & & & 78.0 & 48.6 \\
\hline 4703.26 & 8.01 & 0.21 & 28.511 & & & & & 45.0 & 20.0 & & \\
\hline 4705.32 & 8.01 & 0.57 & 26.248 & 57.0 & 57.5 & 119.0 & & 102.0 & 47.8 & 104.0 & \\
\hline 4709.99 & 8.01 & -0.47 & 26.224 & & & 58.0 & & 59.0 & 22.8 & 44.0 & \\
\hline 4906.82 & 8.01 & -0.03 & 26.303 & & & 96.0 & 82.0 & 52.0 & 16.3 & 64.0 & \\
\hline 4941.11 & 8.01 & 0.08 & 26.552 & & & & & 54.0 & 23.5 & & \\
\hline 4943.00 & 8.01 & 0.37 & 26.560 & & 45.0 & & & 63.0 & 30.5 & 68.0 & \\
\hline
\end{tabular}


Table A.3. Equivalent widths of the lines in spectra of stars of NGC 6231.

\begin{tabular}{|c|c|c|c|c|}
\hline & & & & \\
\hline Wave & code & $\log g f$ & $E_{\text {low }}, \mathrm{eV}$ & 161 \\
\hline 4009.26 & 2.00 & -1.47 & 21.217 & \\
\hline 4143.76 & 2.00 & -1.19 & 21.217 & \\
\hline 4437.55 & 2.00 & -2.03 & 21.217 & 33.0 \\
\hline 4713.14 & 2.00 & -1.23 & 20.963 & \\
\hline 3920.68 & 6.01 & -0.21 & 16.332 & 44.0 \\
\hline 4374.28 & 6.01 & 0.66 & 24.652 & \\
\hline 5137.26 & 6.01 & -0.93 & 20.700 & \\
\hline 5139.17 & 6.01 & -0.74 & 20.703 & \\
\hline 5143.49 & 6.01 & -0.24 & 20.703 & \\
\hline 5145.16 & 6.01 & 0.16 & 20.709 & \\
\hline 5151.09 & 6.01 & -0.20 & 20.709 & \\
\hline 3955.85 & 7.01 & -0.78 & 18.465 & \\
\hline 3995.00 & 7.01 & 0.28 & 18.496 & \\
\hline 4035.08 & 7.01 & 0.62 & 23.123 & \\
\hline 4041.31 & 7.01 & 0.85 & 23.141 & \\
\hline 4043.53 & 7.01 & 0.74 & 23.131 & \\
\hline 4073.05 & 7.01 & -0.16 & 23.123 & \\
\hline 4082.27 & 7.01 & 0.15 & 23.131 & \\
\hline 4171.59 & 7.01 & 0.28 & 23.195 & \\
\hline 4176.16 & 7.01 & 0.60 & 23.195 & \\
\hline 4199.98 & 7.01 & 0.03 & 23.245 & \\
\hline 4227.74 & 7.01 & -0.07 & 21.598 & \\
\hline 4237.05 & 7.01 & 0.55 & 23.241 & \\
\hline 4241.79 & 7.01 & 0.71 & 23.245 & \\
\hline 4432.74 & 7.01 & 0.58 & 23.414 & \\
\hline 4447.03 & 7.01 & 0.29 & 20.408 & \\
\hline 4530.41 & 7.01 & 0.67 & 23.473 & \\
\hline 4601.48 & 7.01 & -0.37 & 18.465 & \\
\hline 4607.15 & 7.01 & -0.49 & 18.461 & \\
\hline 4613.87 & 7.01 & -0.73 & 18.465 & \\
\hline 4621.39 & 7.01 & -0.54 & 18.465 & \\
\hline 4630.54 & 7.01 & 0.13 & 18.482 & 116.0 \\
\hline 4643.09 & 7.01 & -0.34 & 18.482 & \\
\hline 4779.72 & 7.01 & -0.56 & 20.645 & \\
\hline 4788.14 & 7.01 & -0.38 & 20.652 & \\
\hline 4803.29 & 7.01 & -0.12 & 20.664 & \\
\hline 5002.70 & 7.01 & -1.02 & 18.461 & \\
\hline 5005.15 & 7.01 & 0.61 & 20.664 & \\
\hline 5007.33 & 7.01 & 0.16 & 20.939 & \\
\hline 5010.62 & 7.01 & -0.52 & 18.465 & \\
\hline 5025.66 & 7.01 & -0.44 & 20.664 & \\
\hline 5045.10 & 7.01 & -0.33 & 18.482 & \\
\hline 3945.04 & 8.01 & -0.69 & 23.418 & \\
\hline 3982.72 & 8.01 & -0.67 & 23.440 & \\
\hline 4062.91 & 8.01 & -0.09 & 28.704 & \\
\hline 4071.24 & 8.01 & -0.09 & 28.692 & \\
\hline 4072.16 & 8.01 & 0.53 & 25.648 & \\
\hline 4075.87 & 8.01 & 0.69 & 25.664 & \\
\hline 4078.86 & 8.01 & -0.26 & 25.637 & \\
\hline 4083.93 & 8.01 & 0.15 & 28.682 & \\
\hline 4085.12 & 8.01 & -0.14 & 25.648 & \\
\hline 4087.20 & 8.01 & 0.53 & 28.675 & \\
\hline 4092.93 & 8.01 & -0.25 & 25.664 & \\
\hline 4119.22 & 8.01 & 0.48 & 25.847 & \\
\hline 4129.31 & 8.01 & -1.12 & 25.836 & \\
\hline 4132.79 & 8.01 & -0.07 & 25.830 & \\
\hline 4153.28 & 8.01 & 0.08 & 25.836 & \\
\hline 4156.52 & 8.01 & -0.79 & 25.847 & \\
\hline 4185.44 & 8.01 & 0.71 & 28.356 & \\
\hline 4189.79 & 8.01 & 0.80 & 28.359 & \\
\hline 4275.53 & 8.01 & 0.76 & 28.856 & 76.0 \\
\hline 4294.87 & 8.01 & 0.36 & 28.829 & \\
\hline 4313.42 & 8.01 & 0.46 & 28.882 & \\
\hline 4317.15 & 8.01 & -0.32 & 22.965 & \\
\hline 4319.64 & 8.01 & -0.32 & 22.978 & \\
\hline 4325.76 & 8.01 & -1.06 & 22.965 & \\
\hline 4366.91 & 8.01 & -0.24 & 22.998 & \\
\hline 4369.31 & 8.01 & -0.35 & 26.224 & \\
\hline 4414.88 & 8.01 & 0.17 & 23.440 & 79.0 \\
\hline 4416.97 & 8.01 & 0.04 & 23.418 & 85.0 \\
\hline 4443.04 & $\begin{array}{l}0.01 \\
8.01\end{array}$ & 0.00 & 28.356 & \\
\hline 4448.34 & 8.01 & 0.13 & 28.359 & \\
\hline 4452.38 & 8.01 & -0.74 & 23.440 & \\
\hline 4591.01 & 8.01 & 0.45 & 25.660 & \\
\hline 4596.20 & 8.01 & 0.29 & 25.660 & \\
\hline 4602.06 & 8.01 & 0.51 & 29.061 & \\
\hline 4609.37 & 8.01 & 0.67 & 29.067 & \\
\hline 4610.17 & 8.01 & -0.17 & 29.061 & \\
\hline 4638.86 & 8.01 & -0.30 & 22.965 & \\
\hline 4649.14 & 8.01 & 0.31 & 22.998 & \\
\hline 4661.64 & 8.01 & -0.17 & 22.978 & 60.0 \\
\hline 4673.75 & 8.01 & -1.07 & 22.978 & \\
\hline 4676.24 & 8.01 & -0.30 & 22.998 & 36.0 \\
\hline 4696.36 & 8.01 & -1.31 & 22.998 & \\
\hline 4699.19 & 8.01 & 0.37 & 28.508 & \\
\hline 4703.26 & 8.01 & 0.21 & 28.511 & \\
\hline 4705.32 & 8.01 & 0.57 & 26.248 & \\
\hline 4709.99 & 8.01 & -0.47 & 26.224 & 92.0 \\
\hline 4906.82 & 8.01 & -0.03 & 26.303 & \\
\hline 4941.11 & 8.01 & 0.08 & 26.552 & \\
\hline 4943.00 & 8.01 & 0.37 & 26.560 & \\
\hline 4955.74 & 8.01 & -0.42 & 26.560 & \\
\hline
\end{tabular}




\section{References}

Ahumada, J., \& Lapasset, E. 1995, A\&A, 109, 375

Andrievsky, S. M., Korotin, S. A., Luck, R. E., \& Kostynchuk, L. Yu. 1999, A\&A, 342, 756

Arp, H. C., \& van Sant, C. T. 1958, AJ, 63, 431

Auer, L. H., \& Mihalas, D. 1973, ApJS, 25, 433

Balona, L. A. 1994, MNRAS, 267, 1060

Balona, L. A., \& Koen, C. 1994, MNRAS, 267, 1071

Balona, L. A., \& Laney, C. D. 1995, MNRAS, 276, 627

Carlsson, M. 1986, Uppsala Obs. Rep. 33

Cunha, K., \& Lambert, D. L. 1994, ApJ, 426, 170

Daflon, S., Cunha, K., \& Becker, S. R. 1999, ApJ, 522, 950

Dimitrov, D. L., \& Kubat, J. 1988, Bull. Astron. Inst. Czech. 39, 265

Dufton, P. L. 1972, A\&A, 16, 301

Feast, M. W. 1958, MNRAS, 118, 618

Feast, M. W. 1963, MNRAS, 126, 11

Garcia, B., \& Mermilliod, J. C. 2001, A\&A, 368, 122

Gies, D. R., \& Lambert, D. L. 1992, ApJ, 387, 673

Grevesse, N., Noels, A., \& Sauval, J. 1996, Standard Abundances, in Cosmic Abundances, ed. S. S. Holt, \& G. Sonneborn, ASP Conf. Ser., 99, 117

Groenewegen, M. A. T., \& Lamers, H. J. G. L. M. 1989, A\&AS, 79,359

Heger, A., \& Langer, N. 2000, ApJ, 544, 1016

Holweger, H. 2001, in Solar and Galactic composition, ed. R. F. Wimmer-Schweingruber (Berlin: Springer), in press

Hou, J. L., Prantzos, N., \& Boissier, S. 2000, A\&A, 362, 921

Howarth, I. D., \& Smith, K. C. 2001, MNRAS, 327, 353

Hubeny, I., Lanz, T., \& Jeffery, C. S. 1994, Newslett. Analysis Astron. Spectra, 20, 30

King, D. S. 1981, Sidney Obs. Papers, 89

Korotin, S. A., Andrievsky, S. M., \& Kostynchuk, L. Yu. 1999a, ApSS, 260, 531

Korotin, S. A., Andrievsky, S. M., \& Kostynchuk, L. Yu. 1999b, A\&A, 342, 756

Korotin, S. A., Andrievsky, S. M., \& Luck, R. E. 1999, A\&A, 351,168

Kudritzki, R. P. 1992, A\&A, 266, 395

Kurucz, R. L. 1992, Model atmospheres for population synthesis, in The Stellar Populations of Galaxies, ed. B. Barbuy, \& A. Renzini (Dordrecht: Kluwer), IAU Symp., 149, 225
Kurucz, R. L. 1994, CD-ROM 23

Lamers, H. J. 1972, A\&A, 17, 34

Lamers, H. J. G. L. M., \& Achmad, L. 1994, A\&A, 291, 856

Lennon, D. J., \& Dufton, P. L. 1986, A\&A, 155, 79

Levato, H., \& Malaroda, S. 1980, PASP, 92, 323

Luck, R. E., Andrievsky, S. M., Kovtyukh, V. V., Korotin, S. A., \& Beletsky, Yu. V. 2000, A\&A, 361, 189

Lyubimkov, L. S. 1984, Astrofiz., 20, 255

Lyubimkov, L. S. 1989a, Astrofiz., 29, 704

Lyubimkov, L. S. 1989b, Astrofiz., 30, 58

Maeder, A. 1987, A\&A, 178, 159

Maeder, A., \& Meynet, G. 1988, A\&AS, 76, 411

McCarthy, J. K., Kudritzki, R. P., Lennon, D. J., Venn, K. A., \& Puls, J. 1997, ApJ, 482, 757

McErlean, N. D., Lennon, D. J., \& Dufton, P. L. 1998, A\&A, 329,613

Mermilliod, J.-C. 1999, WEBDA database, obswww . unige.ch/webda/

Moon, T. T., \& Dworetsky, M. M. 1985, MNRAS, 217, 305

Morgan, W. W., Code, A. D., \& Whitford, A. E. 1955, ApJS, 2,41

Napiwotzki, R. 1994, priv. communication

Prieto, C. A., Lambert, D. L., \& Apslund, M. 2001, ApJ, 556, L63

Rolleston, W. R. J., Smartt, S. J., Dufton, P. L., \& Ryans, R. S. I. 2000, A\&A, 363, 537

Schaller, G., Schaerer, D., Meynet, G., \& Maeder, A. 1992, A\&AS, 96, 269

Schild, R. E. 1970, ApJ, 161, 855

Seggewiss, W. 1968, Veröff. Astron. Univ. Bonn, No. 79

Smartt, S. J., Dufton, P. L., \& Lennon, D. J. 1997, A\&A, 326, 763

Snow, T. P. 2000, J. Geophys. Res., 105, 239

Struve, O. 1944, ApJ, 100, 189

Turner, D. G., Grieve, G. R., Herbst, W., \& Harris, W. E. 1980, AJ, 85, 1193

Van Helden, R. 1972, A\&A, 21, 209

Vrancken, M., Lennon, D. J., Dufton, P. L., \& Lambert, D. L. 2000, A\&A, 358, 639

Walborn, N. R. 1972, AJ, 77, 312 